\title{
miRNA-138 regulates MLK3/JNK/MAPK pathway to protect BV-2 cells from $\mathrm{H}_{2} \mathrm{O}_{2}$-induced apoptosis
}

\author{
Ren R, Chen SD, Fan J, Zhang G, Li JB \\ Neurocritical care unit, the second affiliated hospital, Zhejiang University College of Medicine, \\ Hangzhou, Zhejiang Province, P.R. China. goldentongli@zju.edu.cn
}

\begin{abstract}
BACKGROUND: miR-138 is one of the down-regulated miRNAs during acute spinal cord injury. Mixed lineage kinase 3 (MLK3), a key factor of jun N-terminal kinase (JNK)/mitogen-activated protein kinase (MAPK) pathway, is the target of miR-138. The aim of this study was to investigate the role of miR-138 in $\mathrm{H}_{2} \mathrm{O}_{2}$-treated BV-2 cells. METHODS: Murine microglia BV-2 cells were treated with $\mathrm{H}_{2} \mathrm{O}_{2}$ and tested for cell viability and miR-138 expression. The cells were then transfected with miR-138 agomir or miR-138 antagomir, and treated with $200 \mu \mathrm{M}$ $\mathrm{H}_{2} \mathrm{O}_{2}$ for $24 \mathrm{~h}$. The cellular apoptosis was detected by Aennexin V/PI staining. Expression of miR-138, MLK3, and other factors of JNK/MAPK pathway was detected.

RESULTS: After treatment of various concentrations of $\mathrm{H}_{2} \mathrm{O}_{2}$, the cell viabilities were reduced, and miR-138 expression was down-regulated. Compared to the control cells, over-expressing miR-138 in BV-2 cells reduced apoptosis rate from $24.2 \%$ to $11.9 \%$. Western blot further showed that JNK, p-JNK, c-jun, p-c-jun, p38 MAPK, and p-p38 MAPK were down-regulated. Expression of pro-apoptosis factors iNOS and COX-2 were also downregulated. Transfection of miR-138 antagomir produced the opposite effect of the transfection of miR-138 agomir. CONCLUSION: miR-138 was able to reduce $\mathrm{H}_{2} \mathrm{O}_{2}$-induced apoptosis in $\mathrm{BV}-2$ cells. The protective effect was related to the down-regulation of MLK3 proteins and sequentially inhibiting JNK/MAPK signaling pathway (Fig. 3, Ref. 27). Text in PDF www.elis.sk.

KEY WORDS: miR-138, mixed lineage kinase 3, apoptosis, microglia, jun $\mathrm{N}$-terminal kinase.
\end{abstract}

\section{Introduction}

Spinal cord injury (SCI) refers to the spinal cord damage caused by trauma, diseases, or degeneration. A recent review demonstrated that traffic accidents and fall in the elderly population are the top two causes of SCI (1). Considering currently limited treatment methods and the rising aging population, SCI not only causes serious physical and psychological harms to patients, but also brings heavy economic and social burden (2). The study of the pathological changes in SCI can play a positive role in understanding the molecular mechanism of the injury, finding new drug targets, and improving the effect of interventions.

MicroRNAs (miRNAs) are the group of endogenous small non-coding RNAs that negatively regulate gene expression (3). MiRNAs are present in all kinds of systems, including the central nervous system, and their dysregulation was related to the multiple neurotraumatic diseases (4). Recently, the role of the miRNAs in SCI development and their value in SCI diagnosis were reported in several papers (5-7). The microarray analysis revealed that miR138 expression was significantly enriched in the adult rat spinal

Neurocritical care unit, the second affiliated hospital, Zhejiang University College of Medicine, Hangzhou, Zhejiang Province, P.R. China

Address for correspondence: Jingbo Li, Neurocritical care unit, the second affiliated hospital, Zhejiang University College of Medicine, 88 Jiefang Road, Hangzhou 310009, Zhejiang Province, P.R. China

Phone: +86-13588773152, Fax: +86.571.87783777 cord following SCI (8). Mixed-lineage kinase 3 (MLK3) contains seeding sequences of miR-138 in its 3'-untransltaed region (UTR) and was proved to be a direct target of miR-138 (9). MLK3 is a member of mitogen-activated protein kinase (MAPK) family and it can activate jun N-terminal kinase (JNK) signaling pathway via phosphorylating MAP2K members (10).

JNK signaling is one of the three major MAPK pathways and is a well-known regulator under oxidative stress (11). During SCI, oxidative conditions can increase expression of inducible nitric oxide synthase (iNOS) and trigger release of nitric oxide (NO) free radicals (12). JNK/MAPK signaling pathway is involved in oxidative stress-induced NO production and contributes to cytokine-induced apoptosis $(13,14)$. In the central nervous system, microglia cells play a role of resident macrophages and they are activated in response to brain injury (15). Apoptosis of microglia cells is responsible for neuronal degeneration in many diseases of central nervous system (16). Based on the previous studies, we inferred that miR-138 might play a protective role via regulating JNK/MAPK signaling and we tested this hypothesis in peroxide microglia cells.

\section{Materials and methods}

\section{Chemicals and reagents}

Dulbecco's Modified Eagles Medium (DMEM), fetal bovine serum (FBS), penicillin, and streptomycin were purchased from HyClone (GE Healthcare, Logan, USA). Nucleotides used for 
transfection and qPCR were designed and synthesized by GenePharm Co. Ltd. (Shanghai, China). The antibodies used in western blot were purchased from Abcam (Cambridge, UK).

\section{Cell culture}

Murine microglia BV-2 cells (ATCC, Manassas, USA) were cultured with DMEM supplemented with $10 \%$ FBS, $100 \mathrm{U} / \mathrm{ml}$ penicillin, and $100 \mu \mathrm{g} / \mathrm{ml}$ streptomycin. The cells were cultured at $37{ }^{\circ} \mathrm{C}$ in a humidified incubator with $5 \% \mathrm{CO}_{2}$. Prior to each assay, cells were inoculated in 6-well plates at a density of $2 \times 10^{5}$ cells and cultured for 2 days to reach synchronization (70-80\% confluence).

\section{Induction of oxidative injury}

To induce oxidative injury, BV-2 cells ( $80 \%$ confluence) were treated with various concentrations of $\mathrm{H}_{2} \mathrm{O}_{2}(100 \mu \mathrm{M}, 200 \mu \mathrm{M}$, $400 \mu \mathrm{M}$ ) for $24 \mathrm{~h}$. The cells were then tested for cell viability by Enhanced Cell Counting Kit-8 (Beyotime Biotechnology, Shanghai, China) as instructed. The harvested cells were also tested for expression of miR-138.

\section{Cell transfection}

For over-expression of miR-138, BV-2 cells were transfected with 100 pmol of miR-138 agomir or agomir-negative control (agomir-NC). To inhibit miR-138 expression, BV-2 cells were transfected with 100 pmol of miR-138 antagomir or antagomirnegative control (antagomir-NC). All transfections were performed using Lipofectamine ${ }^{\mathrm{TM}} 2000$ (Thermo Fisher Scientific, Inc., Carlsbad, USA) following manufacturer's instructions.

After $48 \mathrm{~h}$ of transfection, the cells were treated with $200 \mu \mathrm{M}$ $\mathrm{H}_{2} \mathrm{O}_{2}$ and cultured for another $24 \mathrm{~h}$. Meanwhile, BV-2 cells without transfection were treated with hydrogen peroxide and taken as the peroxide control; BV-2 cells cultured as normal were used as normoxia control. At the end of $\mathrm{H}_{2} \mathrm{O}_{2}$ treatment, the cells were harvested for further assays.

\section{Cellular apoptosis detection}

Cellular apoptosis was measured using Annexin V-FITC/PI apoptosis assay kit (Sangon Biotech, Shanghai, China). Cells were harvested as described above, washed with PBS, and suspended in binding buffer. The cells were then stained with Annexin V-FITC and propidium iodide solution. After incubation for $15 \mathrm{~min}$ in the dark at room temperature, the cell suspensions were analyzed on CytoFlex flow cytometer (Beckman-Coutler Inc., Brea, USA).

\section{Real-time quantitative PCR}

Total RNA was extracted from cells using miRNeasy mini kit (Qiagen, Hilden, Germany). The quality and concentration of RNA samples were measured using the NanoDrop 1000 (ThermoFisher Scientific, Waltham, USA). Complementary DNA (cDNA) was synthesized from RNA using PrimeScript RT Master Mix reagent kit (Takara Biotechnology, Co., Ltd., Dalian, China). The primer sequences used for reverse transcription were as follows: miR-138, 5'-GTCGTATCCAGTGCAGGGTCCGAGGTATTCGCACTGGATACGACTCGACCA-3'; U6, 5'-AAAATATGGAACGCT-3'.
The oligo dT primer was used for reverse transcription of MLK3. qPCR was performed using the SYBR premix EX Taq kit (Takara Biotechnology, Co., Ltd.) on GeneAmp PCR System 9700 (Applied Biosystem, Foster, USA). Standard thermocycling conditions were used: $95^{\circ} \mathrm{C}$ for $30 \mathrm{~s} ; 40$ cycles of $95^{\circ} \mathrm{C}$ for $5 \mathrm{~s}$ and $60^{\circ} \mathrm{C}$ for $30 \mathrm{~s}$. U6 and glyceraldehyde-3-phosphate dehydrogenase (GAPDH) were used as control of miR-138 and MLK3 mRNA, respectively. The primers used for qPCR were as followed:5'-TCCGAGCCTGACTAAGTGTTGTGGTCGA-3' and 5'-GTGCAGGGTCCGAGGT-3' for miR-138; 5'-AGCAAACTCCGAGCAAGGGAC-3' and 5'-GGCTAAACCAGAACTCAAGCGTG-3' for MLK3; 5'-TCCGATCGTGAAGCGTTC-3' and 5'-GTGCAGGGTCCGAGGT-3' for U6; 5'-GGATTTGGTCGTATTGGG-3' and 5'-GGAAGATGGTGATGGGAT-3' for GAPDH. The relative expressions of miR138 and MLK3 were normalized to U6 or GAPDH expression using the $2^{-\Delta \Delta \mathrm{Ct}}$ method (17).

\section{Western blot}

The cells were harvested and then lysed in RIPA lysis buffer (Boster Biological Technology, Wuhan, China) to prepare total protein lysates. Protein $(50 \mu \mathrm{g} / \mathrm{lane})$ was separated by $10 \%$ SDSpolyacrylamide gel, electroblotted onto a nitrocellulose membrane, and blocked with $5 \%$ non-fat milk. The membrane was incubated with specific primary antibodies overnight at $4{ }^{\circ} \mathrm{C}$ and incubated with appropriate secondary antibodies for $1 \mathrm{~h}$ at room temperature. Antibodies were used at 1:1000 dilutions as followed: rabbit monoclonal anti-JNK1/JNK2/JNK3 (ab179461), rabbit monoclonal anti-JNK1/JNK2/JNK3 (phospho T183/T182/ T221, ab124956), rabbit monoclonal anti-c-jun (ab32137), rabbit polyclonal anti-c-jun (phosphor S73, ab30620), rabbit monoclonal anti-p38 (ab170099), rabbit polyclonal anti-p38 (phosphor Y182, ab47363), rabbit monoclonal anti-MLK3 (ab51068), rabbit polyclonal anti-iNOS (ab3523), rabbit polyclonal anti-COX2 (ab102005), rabbit polyclonal anti-GAPDH (ab9485), goat antirabbit IgG (horseradish peroxidase-conjugated, ab205718). Immunodetection was measured using the enhanced chemiluminescence kit (Beyotime Biotechnology, Shanghai, China).

\section{Statistics}

The statistical analyses were performed using the SPSS 21.0 software. The quantitative data were presented as the mean \pm standard deviation (SD). Comparisons between two independent groups were determined by $t$-test. Comparisons among multiple groups were determined by one-way ANOVA with Tukey's test. A $\mathrm{p}<0.05$ was designated as statistically significant. All the experiments were repeated at least three times and representative results were exhibited in this study.

\section{Results}

\section{Expression of miR-138 in peroxide microglia cells}

Firstly, we tested whether the expression of miR-138 was altered when BV-2 cells were treated with hydrogen peroxide. As shown in Figure 1, peroxide conditions reduced cell viability and inhibited miR-138 expression. When BV-2 cells were treated with 
(A)

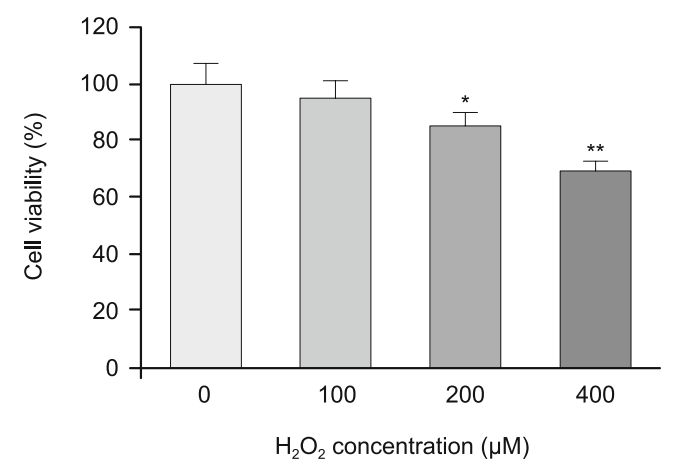

B

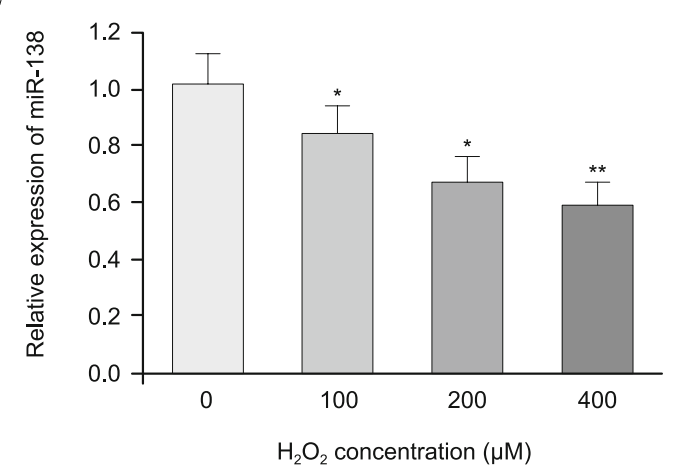

Fig. 1. Hydrogen peroxide inhibits cell viability and reduces miR-138 expression. BV-2 cells were treated with various concentrations of $\mathrm{H}_{2} \mathrm{O}_{2}$ for $24 \mathrm{~h}$ and collected for detection. Cells without $\mathrm{H}_{2} \mathrm{O}_{2}$ were taken as the control. A. Cell viability tested by CCK-8 method. B. Relative expression of miR-138 tested by RT-PCR. * $p<0.05$ vs control. ** p $<0.01$ vs control.

$200 \mu \mathrm{M} \mathrm{H}_{2} \mathrm{O}_{2}$, cell viability was reduced to $85.4 \%$ of the control cells $(\mathrm{p}<0.05)$. Meanwhile, expression of miR-138 was also significantly inhibited compared to the control $(\mathrm{p}<0.05)$. Treating with $400 \mu \mathrm{M} \mathrm{H}_{2} \mathrm{O}_{2}$ further suppressed cell viability and miR-138 expression. Although treating with $100 \mu \mathrm{M} \mathrm{H}_{2} \mathrm{O}_{2}$ could also inhibit miR-138 expression, cell viability was not significantly affected ( $p>0.05$ ). Therefore, we selected $200 \mu \mathrm{M}$ as the treatment concentration of $\mathrm{H}_{2} \mathrm{O}_{2}$ in the following assays.

Effect of miR-138 on cellular apoptosis under peroxide conditions

SCI-induced oxidative stress is an important cause of cellular apoptosis. Next, we tested the effect of miR-138 on apoptosis of murine microglia BV-2 cells treated with $\mathrm{H}_{2} \mathrm{O}_{2}$. As shown in Figure 2, treating with $200 \mu \mathrm{M} \mathrm{H}_{2} \mathrm{O}_{2}$ increased apoptotic rate of BV-2 cells (peroxide vs. normoxia). When over-expressing miR-138 in BV-2 cells, apoptotic rate significantly decreased from $24.2 \%$ to $11.9 \%$ $(\mathrm{p}<0.01)$, although the rate was still higher than the rate $(4.2 \%)$ in normally cultured cells. On the other hand, inhibiting miR-138 expression in BV-2 cells could aggravate cellular apoptosis $(\mathrm{p}<$ 0.01 ). The results indicated that miR-138 had anti-apoptosis role in peroxide BV-2 cells.
(A)
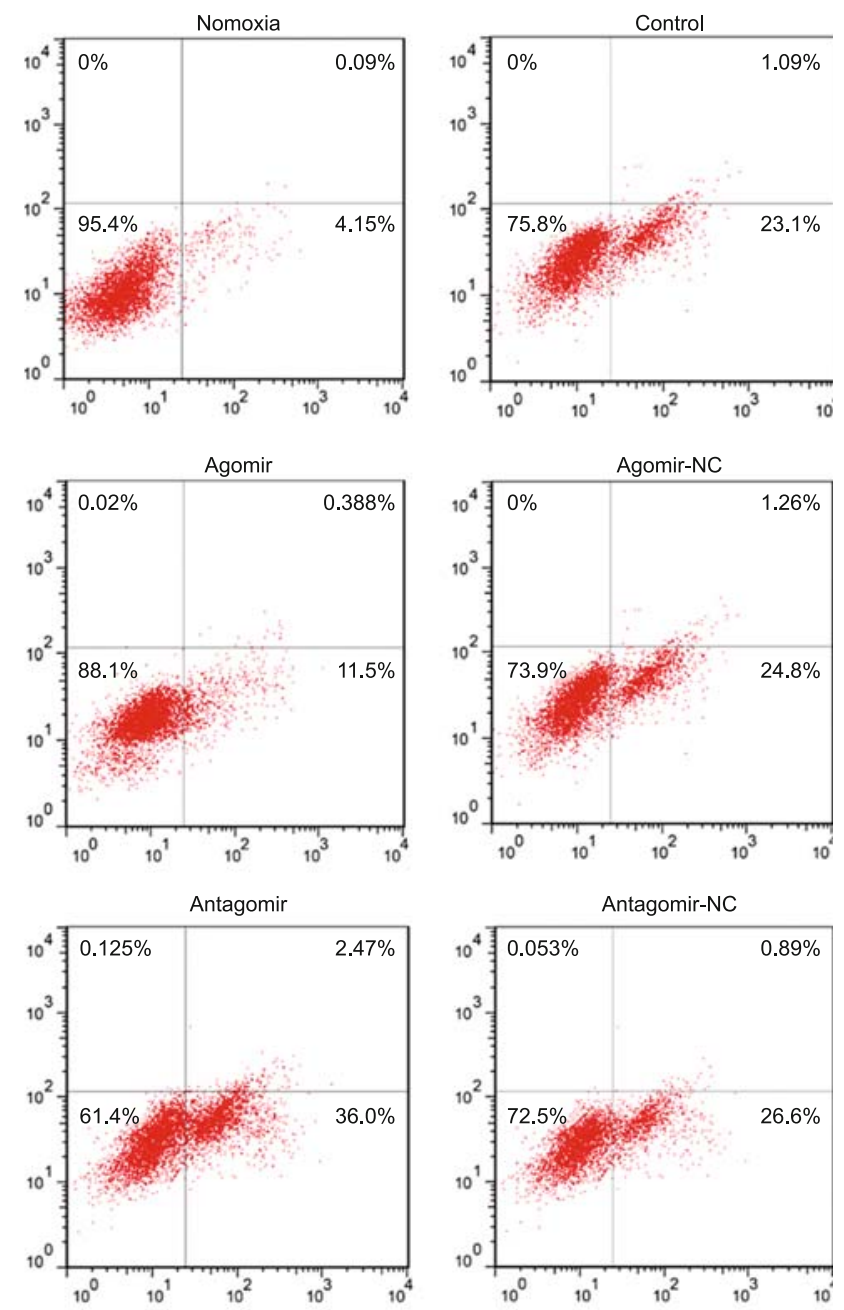

(B)

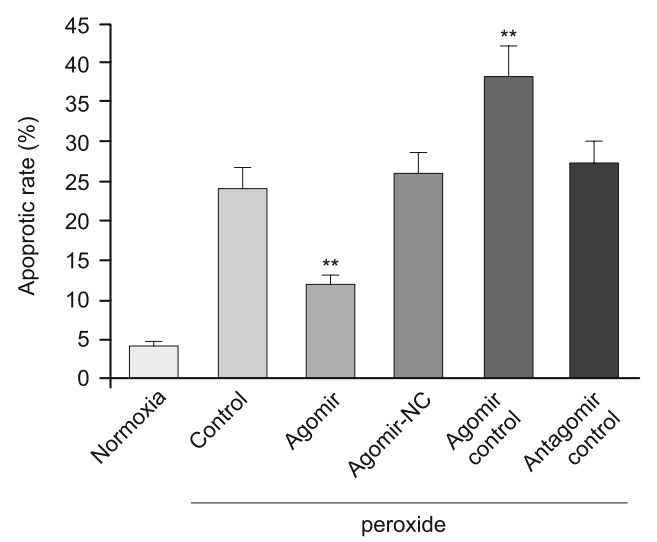

Fig. 2. miR-138 reduces cellular apoptosis under peroxide condition. BV-2 cells were transfected with miR-138 agomir or miR-138 antagomir and then treated with $200 \mu \mathrm{M} \mathrm{H}_{2} \mathrm{O}_{2}$ for $24 \mathrm{~h}$. The cell apoptotic rate was tested by Annexin V/PI staining and flow cytometry (A $\&$ B). Cells without transfection and treated with hydrogen peroxide were taken as the peroxide control; cells cultured as normal were used as normoxia control. ** $\mathrm{p}<\mathbf{0 . 0 1}$ vs peroxide control. 
A

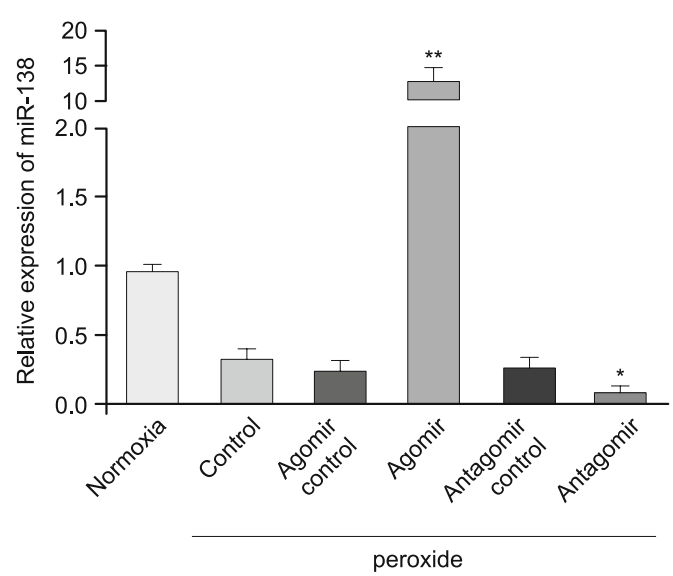

(B)

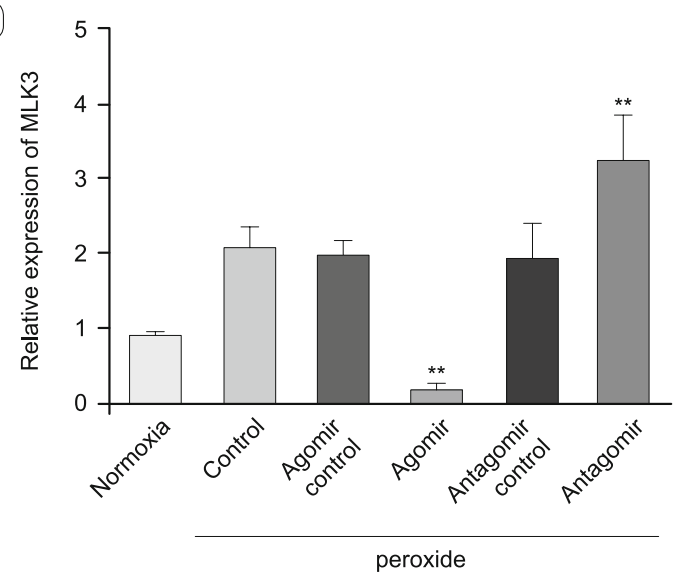

Effect of miR-138 on MLK3/JNK/MAPK pathway in peroxide microglia cells

Because MLK3, the key regulator of JNK/MAPK pathway, is a confirmed target of miR-138 (9), next we investigated whether miR-138 could regulate MLK3/JNK/MAPK signaling in peroxide BV-2 cells. The results of Figure 3A and 3B showed that over-expressing miR-138 led to less expression of MLK3 and inhibiting miR-138 promoted MLK3 expression. As indicated by the Western blot (Figure 3C), peroxide induced expression of MLK3, JNK (total and active form phospho-JNK), c-jun (total and phospho-c-jun), and p38 MAPK (total and phospho-p38), indicating that MLK3/JNK/MAPK was activated under peroxide condition. The activation effect could be eased up by miR-138 agomir and further enhanced by miR-138 antagomir. In addition, we also detected the effect of miR-138 on expression of pro-apoptosis factors iNOS and COX-2. Consistent with the activation of MLK3/JNK/MAPK, peroxide condition and miR-138 antagomir could also induce expression of iNOS and COX-2, while miR-138 agomir inhibited expression of these two proteins. The results indicated that miR-138 played an anti-apoptosis role via suppressing MLK3/JNK/MAPK signaling.
C

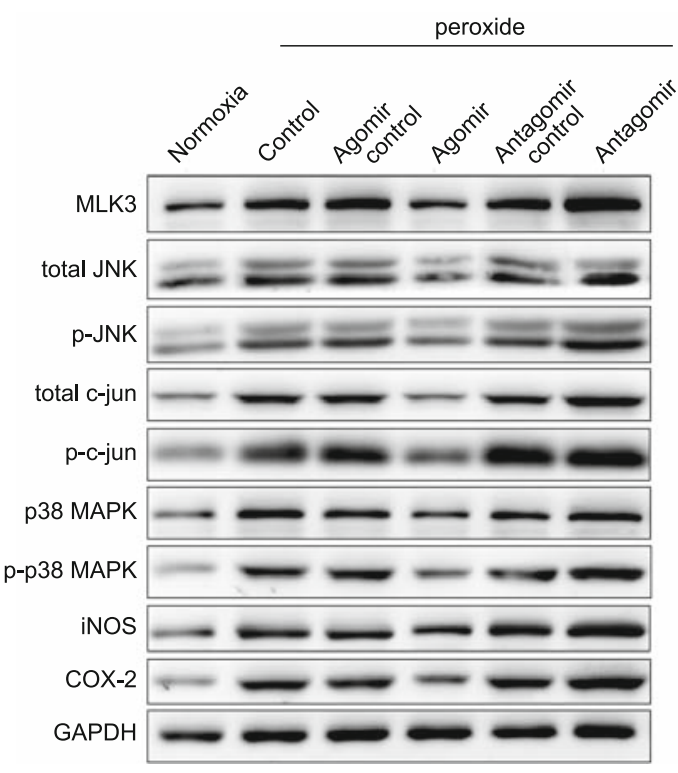

Fig. 3. miR-138 regulates MLK3 expression and inhibits activity of JNK/MAPK signaling pathway. BV-2 cells were transfected with miR138 agomir or miR-138 antagomir and then treated with $200 \mu \mathrm{M} \mathrm{H}_{2} \mathrm{O}_{2}$ for $24 \mathrm{~h}$. Cells without transfection and treated with hydrogen peroxide were taken as the peroxide control; cells cultured as normal were used as normoxia control. A) Relative expression of miR-138 normalized to U6 in various groups. B) Relative expression of MLK3 normalized to GAPDH in various groups. C) Protein levels of MLK3 and key factors of JNK/MAPK pathway tested by western blot. GAPDH was used as internal control. * $\mathrm{p}<0.05$ vs peroxide control; ** $\mathrm{p}<0.01$ vs peroxide control.

\section{Discussion}

It is well known that primary and secondary injuries during SCI cause devastating health problems in thousands of patients every year (18). Secondary lesions could be reversed and modulated; hence this has been a hot research topic of SCI (19). The oxidative stress and NO production triggered by SCI is an important pathophysiological process during the secondary injury. Excessive reactive oxygen species lead to severe tissue damage and neuronal death and affect the repair and function recovery of neurons after SCI (20). In this study, we showed that miR-138 could protect microglia BV-2 cells from $\mathrm{H}_{2} \mathrm{O}_{2}$-induced apoptosis through inhibiting MLK3/JNK/MAPK signaling pathway.

In this study, we identified MLK3 as a novel target of miR138. MLK3 is a well- known regulator of JNK by phosphorylation; activated JNK subsequently phosphorylated transcription factor cjun and initiated expression of apoptosis-related genes (21). Therefore, MLK3 can be considered as a pro-apoptosis kinase, which was indicated by our results (Figs 2 and 3). Similar pro-apoptosis function of MLK3 was also reported in the previous studies (see review 22). When blocking MLK3 activity by viral protein, RNAi technology, or specific inhibitor, the pro-apoptotic effect could be 
prevented $(23,24)$. In our study, inhibiting MLK3 expression by over-expressing miR-138 also reduced cell apoptosis.

In addition to MLK3, possible targets of miR-138 also include apoptosis-related genes like caspase-3, calpain 1, calpain 2, Bcl-2, $\mathrm{c}-\mathrm{Myc}$, and apoptosis-inducing factor $\operatorname{AIF})(8,25)$. Some of these target genes were reported to be involved in the pathological process of SCI. Activation of caspase-3 was observed during a delayed paraplegia after spinal cord ischemia (26). After contusion-derived SCI, the induced apoptosis of the spinothalamic tract cells was correlated with prompt decrease in anti-apoptotic protein Bcl-2 (27). Therefore, the regulatory effect of miR-138 during SCI is a complex network and its anti-apoptotic role may be explained by multiple mechanisms.

In conclusion, our results showed that miR-138 was able to reduce hydrogen peroxide-induced apoptosis in BV-2 microglia cells. The protective effect might be related to the down-regulation of MLK3 proteins and sequentially inhibiting JNK/MAPK signaling pathway. These findings may provide new insights for understanding and treatment of SCI.

\section{Learning points}

- miR-138 expression was down-regulated, and cell viability was inhibited when murine microglia BV-2 cells were treated with $\mathrm{H}_{2} \mathrm{O}_{2}$.

- Over-expressing miR-138 in BV-2 cells could protect the cells from $\mathrm{H}_{2} \mathrm{O}_{2}$-iuduced apoptosis.

- The protective role of miR-138 was related with suppressing MLK3 expression and sequentially inhibiting JNK/MAPK signaling pathway.

\section{References}

1. Singh A, Tetreault L, Kalsiryan S, Nuori A, Gehlings MG. Global prevalence and incidence of traumatic spinal cord injury. Clin Epidemiol 2014; 6: 309-331.

2. Lee BB, Cripps RA, Fitzharris M, Wing PC. The global map for traumatic spinal cord injury epidemiology: update 2011, global incidence rate. Spinal Cord 2014; 52 (2): 110-116.

3. Bartel DP. MicroRNAs: target recognition and regulatory functions. Cell 2009; 136 (2): 215-233.

4. Ning B, Gao L, Liu R, Liu Y, Zhang NS, Chen ZY. microRNAs in spinal cord injury: potential roles and therapeutic implications. Int J Biol Sci 2014; 10 (9): 997-1006.

5. Hachisuka S, Kamei N, Ujigo S, Miyaki S, Yasunaga Y, Ochi M. Circulating microRNAs as biomarkers for evaluating the severity of acute spinal cord injury. Spinal Cord 2014; 52 (8): 596-600.

6. Li P, Teng ZQ, Liu CM. Extrinsic and intrinsic regulation of axon regeneration by microRNAs after spinal cord injury. Neural Plast 2016; 2016: 1279051.

7. Yang J, Xiong LL, Wang YC et al. Oligodendrocyte precursor cell transplantation promotes functional recovery following contusive spinal cord injury in rats and is associated with altered microRNA expression. Mol Med Rep 2018; 17 (1): 771-782.

8. Yunta M, Nieto-Diaz M, Esteban FJ et al. MicroRNA dysregulation in the spinal cord following traumatic injury. PLoS One 2012; 7 (4): e34534.
9. He S, Liu P, Jian Z, Li J, Zhu Y, Feng Z, Xiao Y. miR-138 protects cardiomyocytes from hypoxia-induced apoptosis via MLK3/JNK/c-jun pathway. Biochem Biophys Res Commun 2013; 441 (4): 763-769.

10. Gallo KA, Johnson GL. Mixed-lineage kinase control of JNK and p 38 MAPK pathways. Nat Rev Mol Cell Biol 2002; 3 (9): 663-672.

11. Yan L, Liu S, Wang $\mathbf{C}$ et al. JNK and NADPH oxidase involved in fluoride-induced oxidative stress in BV-2 microglia cells. Mediators Inflamm 2013; 2013: 895975.

12. Yang JY, Kim HS, Lee JK. Changes in nitric oxide synthase expression in young and adult rats after spinal cord injury. Spinal Cord 2007; 45 (11): 731-738.

13. Storling J, Binzer J, Andersson A et al. Nitric oxide contributes to cytokine-induced apoptosis in pancreatic beta cells via potentiation of JNK activity and inhibition of Akt. Diabetologia 2005; 48 (10): 2039-2050.

14. Wang $C$, Nie $X$, Zhang $Y$ et al. Reactive oxygen species mediate nitric oxide production through ERK/JNK MAPK signaling in HAPI microglia after PFOS exposure. Toxicol Appl Pharmacol 2015; 288 (2): 143-151.

15. Hains BC, Waxman SG. Activated microglia contribute to the maintenance of chronic pain after spinal cord injury. J Neurosci 2006; 26 (16): 4308-4317.

16. Frakes AE, Ferraiuolo L, Haidet-Phillips AM et al. Microglia induce motor neuron death via the classical NF- $\mathrm{kB}$ pathway in amyotrophic lateral sclerosis. Neuron 2014; 81 (5): 1009-1023.

17. Livak KJ, Schmittgen TD. Analysis of relative gene expression data using real-time quantitative PCR and the $2^{-\Delta \Delta C t}$ method. Methods 2001; 25 (4): 402-408.

18. Silva NA, Sousa N, Reis RL, Salgado AJ. From basics to clinical: a comprehensive review on spinal cord injury. Prog Neurobiol 2014; 114: 25-57.

19. Oyinbo CA. Secondary injury mechanisms in traumatic spinal cord injury: a nugget of this multiply cascade. Acta Neurobiol Exp (Wars) 2011; 71 (2): 281-299.

20. Liu D, Ling X, Wen J, Liu J. The role of reactive nitrogen species in secondary spinal cord injury: formation of nitric oxide, peroxynitrite, and nitrated protein. J Neurochem 2002; 75 (5): 2144-2154.

21. Liu J, Lin A. Role of JNK activation in apoptosis: a double-edged sword. Cell Res 2005; 15 (1): 36-42.

22. Mota M, Reeder MK, Chernoff J, Bazenet CE. Evidence for a role of mixed lineage kinases in neuronal spoptosis. J Neurosci 2001; 21 (14): 4949-4957.

23. Amako Y, Igloi Z, Mankouri J et al. Hepatitis C virus NS5A inhibits mixed lineage kinase 3 to block apoptosis. J Biol Chem 2013; 288 (34): $24753-24763$.

24. Sui Z, Fan S, Sniderhan LF et al. Inhibition of mixed lineage kinase 3 prevents HIV-1 Tat-mediated neurotoxicity and monocyte activation. J Immunol 2006; 177 (1): 702-711.

25. Zhao X, Yang L, Hu J, Ruan J. miR-138 might reverse multidrug resistance of leukemia cells. Leuk Res 2010; 34 (8): 1078-1082.

26. Kakinohana M, Kida K, Minamishima S, Atochin DN, Huang PL, Kaneki M, Ichinose F. Delayed paraplegia after spinal cord ischemic injury requires caspase-3 activation in mice. Stroke 2011; 42 (8): 2302-2307.

27. Qiu J, Nesic $\mathbf{O}$, Ye $\mathbf{Z}$ et al. Bcl-xL expression after contusion to the rat spinal cord. J Neurotrauma 2001; 18 (11): 1267-1278.

Received February 2, 2018. Accepted March 1, 2018. 